\title{
Rationale and Design of the Brigham Cohort for psoriasis and psoriatic arthritis registry (COPPAR)
}

\author{
Maria Schneeweiss ${ }^{1,2}$, Joseph F. Merola ${ }^{1,2}$, Elizabeth W. Karlson ${ }^{1}$ and Daniel H. Solomon ${ }^{1,3^{*}}$
}

\begin{abstract}
Background: Psoriasis (PsO) and psoriatic arthritis (PsA) are related conditions with poorly defined transition among them, risk factors for progression, complex treatment algorithms, and biomarkers for treatment response and longterm outcomes. We describe the development of a PsO/PsA registry at an academic medical center.

Methods: We developed a single-center PsO/PsA longitudinal disease registry including biorepository that captures relevant disease markers and treatment choices in a circumscribed population with a defined catchment area. We searched the electronic medical record for patients with visits in the last year for PsO or PsA. They formed the potentially eligible registry population. Baseline patient and provider questionnaires were developed using standardized measures, including demographics, comorbidities, medications, specific disease characteristics, functional status, quality of life, mental health, and resource use. An abbreviated set of items was collected every six month and at visits with treatment changes or disease flares. Biospecimens included blood (serum, plasma, DNA, RNA) and skin biopsy samples, with repeat collections of serum and plasma. Data from the EMR to augment the registry questionnaires are available on all patients.

Discussion: Searching the Brigham EMR system from 2013 through 2014, we found 1694 patients with PsO and 1028 with PsA. Their mean age was 55 years and 53\% were female. Of these 17\% had diabetes, 38\% hyperlipidemia, and 45\% hypertension. The median BMI was 29.6. PsA patients used more systemic prednisone, MTX, and TNF alpha inhibitors $(47 \%, 60 \%$, and $66 \%)$ compared to PsO patients (28\%, 20\% and 21\%). We have collected plasma in 410 patients, DNA RNA in 453 patients. In conclusion, we have developed a PsO/PsA registry to better define longitudinal disease characteristics, perform biomarker studies, and examine treatment trends.
\end{abstract}

Keywords: Psoriasis, Psoriatic arthritis, Disease registry, Biomarkers, Electronic health record

\section{What is the most significant finding of this registry?}

This paper describes the structure and functioning of a new psoriasis and psoriatic arthritis disease registry at a major medical center. Early results showed that registry participants were representative for the population of the catchment area.

\section{What does it mean for dermatologists and their patients}

The psoriasis and psoriatic arthritis disease registry will allow new research on the natural progression of disease, typical treatment pathways and the effectiveness of new treatment on clinical and patient-reported outcomes.

\section{Learning points}

\footnotetext{
*Correspondence: dsolomon@bwh.harvard.edu

'Division of Rheumatology, Department of Medicine of the Brigham and Women's Hospital, Harvard Medical School, 75 Francis Street, Boston, MA 02115, USA

${ }^{3}$ Division of Pharmacoepidemiology, Department of Medicine of the Brigham and Women's Hospital, Harvard Medical School, 75 Francis Street, Boston, MA 02115, USA

Full list of author information is available at the end of the article
}

1) There is a wave of new therapeutic options for chronic skin diseases, particularly complex conditions like psoriatic arthritis

2) In order to better understand how new therapies work in routine care of patients with psoriatic arthritis detailed clinical information is necessary 
3) We established a new psoriatic arthritis registry that is embedded in an academic center with a large population with psoriatic arthritis and substantial clinical expertise

4) The new registry will initially include more than 1000 patients with psoriatic arthritis that are longitudinally followed

5) The registry includes validated instruments for physician and patient reported severity and outcome measures plus biomarker and genetic information to study the effectiveness of new therapies

\section{Background}

Disease registries can fill an important gap for improving the understanding of chronic diseases with various treatment strategies. They capture patients in routine care "real-world" settings and collect information on clinical details, biologic materials, patient reported outcomes, and clinical treatment pathways [1]. These types of observational data stand in contrast to most randomized controlled trials that describe highly selected populations. Registries serve complementary roles to studies based on electronic health records with incomplete outcomes information and sparse biospecimens. For patients with psoriasis $(\mathrm{PsO})$ and psoriatic arthritis (PsA) there are few disease registries in Europe [2,3] and the Americas [4, 5]. Some have clearly defined catchment areas and can link to existing national registries [3], others are more geographically diverse [2]. Despite the existing registries, a recent review paper concludes with a call for more PsA registries with systematic capture of patient reported outcomes and bio-specimens [6]. Furthermore, the development of systemic arthritis in patients with $\mathrm{PsO}$ remains a challenging topic of research; thus, a combined $\mathrm{PsO}$ and PsA registry provides important research opportunities.

In considering the development of a combined $\mathrm{PsO} /$ PsA registry, we considered several research opportunities and challenges. First, disease registry information is critically important to understand the natural course of $\mathrm{PsO}$ and PsA utilizing current treatment paradigms. Treatment options for both conditions have rapidly expanded over the last decade and will continue to broaden. A detailed understanding of how and when patients and their providers decide to transition between treatments is important for improving care; it also allow for comparative effectiveness studies to assess real-world benefits and toxicities. Second, around one-third of patients with $\mathrm{PsO}$ develop systemic inflammatory arthritis consistent with PsA [7]. The predictors of PsO to PsA transition include genetic, environmental, and physical examination findings $[7,8]$, but a deeper understanding of the biology of this transition might open up preventive strategies, where there are currently none [9]. Third, registries allow for capture of varied types of data but additional information on comorbid conditions and medication use can be gleaned from linkage between registry data with electronic medical record and health care claims. Finally, PsO has many different clinical phenotypes, including nail disease, scalp, palmo-plantar, pustular, genital, and inverse (intertriginous). Scalp, nail and inverse psoriasis sub-types are common and are associated with an increased risk of PsA, however response to treatments of these subsets has not been well characterized.

Herein, we describe the development of a singlecenter PsO/PsA longitudinal disease registry including biorepository that captures these elements in a circumscribed population with a defined catchment area. The registry is named COPPAR, COhort for Psoriasis and Psoriatic Arthritis Registry, of the Brigham and Women's Hospital in Boston. The specific goals of the registry are to determine biologic, clinical and environmental predictors of PsA among patients initially presenting with $\mathrm{PsO}$; to identify predictors of treatment response and failure and characterize treatment transitions; to quantify health services utilization and quality of life of patients with $\mathrm{PsO}$ and PsA; and to assess relevant subgroups of patients with specific phenotypes, including non-plaque disease (e.g., nail disease, palmar-plantar, genital, inverse).

\section{Methods}

\section{Patients eligible and included in the registry}

The patients eligible for the COPPAR registry have been seen for PsO or PsA at Brigham and Women's Hospital, a large academic medical center in Boston. The hospital's Center for Skin and Related Musculoskeletal Diseases (SARM), a specialist clinic treating patients with concomitant systemic rheumatic and skin diseases [10], has an extensive referral network within the catchment area making the patient population representative of a Northeastern metropolitan population. Patients that might qualify for the registry are identified by systematically screening the electronic medical record (EMR) system of the hospital using an algorithm previously found to be highly predictive of psoriasis [11, 12]. Since the identified potentially eligible subjects will all have their diagnoses confirmed before entering the registry, we simplified the search algorithm to include patients with at least three diagnoses of PsO (ICD-9-CM code 696.1 or ICD-10 code L40.0) or at least three diagnoses of PsA (ICD-9-CM code 696.0 or ICD-10 code L40.5) who also have had a visit to the hospital from 2013 through 2014 for either diagnosis [13, 14].

Using this search strategy, we identify 2484 potentially eligible subjects who have now been invited to participate in the registry. During the baseline visit the treating physician confirms and records the clinical diagnosis using the CASPAR criteria for PsA [15] or the dermatologist- 
defined diagnosis of PsO $[14,16]$. Patients not meeting these criteria will be excluded from participating in the registry.

\section{Questionnaire development}

The registry questionnaires were developed based on an extensive review of validated instruments. Broad areas of interest in the registry include demographics, comorbidities, medications, severity and activity of $\mathrm{PsO} / \mathrm{PsA}$, functional status, quality of life, physical activity, mental health [17], health resource utilization, and physical examination [18]. We consulted with disease experts, outcomes researchers and experts in questionnaires to confirm the final instrument selection (Table 1).

The following instruments were selected for COPPAR: For determining and recording PsA, CASPAR criteria were applied to potential subjects by a rheumatologist [15]. PsO diagnosis was based on expert dermatologist evaluation and/or a skin biopsy $[14,16]$. The 66/68 tender/swollen joint count was conducted by a Rheumatologist for patients with psoriatic arthritis, as a peripheral joint assessment [19]. Enthesitis was assessed using the LEEDS Enthesitis Index (LEI) [20]. Involvement of dactylitis in the hands and/or feet was documented as absent or present (dactylitis count). Axial involvement (past and current) was documented by the physician and was then assessed by the patient using the Bath Ankylosing Spondylitis Disease Activity Index (BASDAI) [21]. To record quantity and severity of skin lesions we used the Psoriasis Areas Severity Index (PASI) [22], Body Surface Area (BSA) [23, 24], and Physician Static Global Assessment (sPGA) [25, 26].

The clinical outcomes measures included both patient and provider derived scores. In patients with PsA, the Multi-Dimensional Health Assessment Questionnaire (MDHAQ) was used to assess pain and functioning [27]. For evaluating the overall health related quality of life we used the European Quality of Life (EuroQoL) instrument [28]. For a more disease specific assessment of quality of life the Dermatology Life Quality Index (DLQI) was used [29, 30], and for patients with PsA the Psoriatic Arthritis Quality of Life (PsAQoL) questionnaire was additionally recorded [31].

We used a validated patient-derived novel PsO outcomes measure, the Comprehensive Assessment of the Psoriasis Patient (CAPP) [32]. CAPP measures plaque, nail, scalp, inverse, genital and palmo-plantar psoriasis with an equally weighted (1 through 5 ) physician objective measure and patient-derived, patient-reported outcome measures (visual analog scales).

Visual Analog Scales (VAS) were used to determine and document patient-perceived pain (with or without arthritis), Patient Global Assessment (PGA), and Physician Global Assessment (PGA) [19, 33]. We used patient reported assessments of work productivity, fatigue, physical activity and mental health. The Work Productivity and Activity Impairment (WPAI) questionnaire was used to assess work productivity [34, 35], the Functional Assessment of Chronic Illness Therapy (FACIT, version 4) was used to assess patient fatigue [36], and the International Physical Activity Questionnaire (IPAQ) was used to determine patients' physical activity $[37,38]$.

In addition to these validated instruments, we documented past and current use of medications including topical agents. During follow-up visits any changes in medication were recorded, including the reason for the change, be it payment issues/insurance problems, treatment failure or adverse reactions. The questionnaires also assess, socio-demographic status, first diagnosis and a family history of psoriasis (with and/or without arthritis), general health features, life style factors, and health care utilization $[39,40]$.

\section{Registry procedures}

After patients have been identified using the electronic medical records system, they are prioritized and invited to visit the SARM clinic of the Brigham and Women's Hospital. Such visits may coincide with dermatology/rheumatology visits or may be scheduled in addition. Prior to their scheduled clinic visits a research assistant prepares the administrative paperwork (informed consent, biospecimen consent, information materials, prospective visit schedule) and physician and patient questionnaires. Upon informed consent, patients undergo a full examination by their treating physician, fill in the patient questionnaire, have blood drawn, and receive an in person follow-up visit schedule for every six months with an internet based questionnaire follow-up sent at three month time points between in person visits (Fig. 1). Patients are emailed a link to the internet based patient questionnaire generated with REDCap (Research Electronic Data Capture): an encrypted, internet-based, electronic data capture tool in line with HIPPA regulations and developed for data capture in research studies [41].

We will collect the following biospecimens annually and then at other specified time points during follow-up: plasma, serum, DNA, RNA, and peripheral blood mononuclear cells (Table 2). These samples will be distributed in aliquots and stored for future use on biomarker studies. In addition, all subjects with $\mathrm{PsO}$ will be asked for an optional skin biopsy of affected skin. We expect that a meaningful proportion of subjects will consent for skin biopsy. Joint fluid is retained for COPPAR if arthrocentesis is performed for otherwise clinically indicated interventions. Results from ultrasound or other imaging studies are available through the EMR system. 
Table 1 Key items recorded by the COPPAR registry

\begin{tabular}{|c|c|c|}
\hline Variables & Physician Assessed & Patient Assessed \\
\hline \multicolumn{3}{|l|}{ Socio-demographic } \\
\hline Demographics & & $\checkmark$ \\
\hline Age & & $\checkmark$ \\
\hline Education & & $\checkmark$ \\
\hline Ethnicity & & $\checkmark$ \\
\hline \multicolumn{3}{|l|}{ Psoriasis (PsO)/Psoriatic Arthritis (PsA) } \\
\hline First PsO or PsA Diagnosis & & $\checkmark$ \\
\hline Family History of PsO and/or PsA & $\checkmark$ & $\checkmark$ \\
\hline CASPAR & $\checkmark$ & \\
\hline Physician Global (VAS) & $\checkmark$ & \\
\hline \multicolumn{3}{|l|}{ General Health Features } \\
\hline Co-morbidities / Drug Toxicities & $\checkmark$ & $\checkmark$ \\
\hline Cardiovascular Risk Factors & $\checkmark$ & $\checkmark$ \\
\hline Infections/Opportunistic Infections & $\checkmark$ & $\checkmark$ \\
\hline Surgical History & & $\checkmark$ \\
\hline Smoking Status & & $\checkmark$ \\
\hline Alcohol Consumption & & $\checkmark$ \\
\hline Mental health (CESD) & & $\checkmark$ \\
\hline Health Care Utilization & & $\checkmark$ \\
\hline \multicolumn{3}{|l|}{ Medications } \\
\hline Current & $\checkmark$ & $\checkmark$ \\
\hline Past & $\checkmark$ & $\checkmark$ \\
\hline Changes & $\checkmark$ & $\checkmark$ \\
\hline Start & $\checkmark$ & $\checkmark$ \\
\hline Stop/reason & $\checkmark$ & $\checkmark$ \\
\hline Change/reason & $\checkmark$ & $\checkmark$ \\
\hline \multicolumn{3}{|l|}{ Peripheral Joint Assessment } \\
\hline $66 / 68$ tender/swollen joint count & $\checkmark$ & \\
\hline Patient swollen joint assessment (VAS) & & $\checkmark$ \\
\hline \multicolumn{3}{|l|}{ Skin Assessment } \\
\hline Psoriasis Area Severity Index (PASI) & $\checkmark$ & \\
\hline Body Surface Area (BSA) & $\checkmark$ & \\
\hline Physician Static Global Assessment (sPGA) & $\checkmark$ & \\
\hline \multicolumn{3}{|l|}{ Pain } \\
\hline Patient tender joint assessment (VAS) & $\checkmark$ & $\checkmark$ \\
\hline \multicolumn{3}{|l|}{ Patient Global } \\
\hline VAS & & $\checkmark$ \\
\hline \multicolumn{3}{|l|}{ Pain and Function } \\
\hline Multi-Dimensional Health Assessment Questionnaire (MDHAQ) & & $\checkmark$ \\
\hline \multicolumn{3}{|l|}{ Health-related quality of life } \\
\hline European Quality of Life assessment (EuroQol) & & $\checkmark$ \\
\hline Dermatology Life Quality Index (DLQI) & & $\checkmark$ \\
\hline Psoriatic Arthritis Quality of Life (PsAQoL) & & $\checkmark$ \\
\hline
\end{tabular}
Enthesitis 
Table 1 Key items recorded by the COPPAR registry (Continued)

\begin{tabular}{lc}
\hline LEEDS Enthesitis Index (LEI) & $\checkmark$ \\
Dactylitis & $\checkmark$ \\
Absent/Present & $\checkmark$ \\
Spinal assessment & \\
Absent/ Present & \\
Bath Ankylosing Spondylitis Disease Activity Index (BASDAl) & $\checkmark$ \\
Psoriasis Assessment & $\checkmark$ \\
CAPP - Plaque Psoriasis & $\checkmark$ \\
CAPP - Scalp Psoriasis & $\checkmark$ \\
CAPP - Nail Psoriasis & $\checkmark$ \\
CAPP - Inverse & $\checkmark$ \\
CAPP - Palm/Sole & $\checkmark$ \\
CAPP - Genital & $\checkmark$ \\
Work Productivity & $\checkmark$ \\
Work Productivity and Activity Impairment (WPAI) & $\checkmark$ \\
Fatigue & \\
Functional Assessment of Chronic IIIness Therapy (FACIT-4) & $\checkmark$ \\
Physical Activity & \\
International Physical Activity Questionnaire (IPAQ) & \\
\hline
\end{tabular}

To ensure high quality data in the registry, the following procedures are followed. Patient questionnaires are reviewed by trained research staff with patients still present to clarify responses. As well, scanners can read all questionnaires reducing human data entry error.

\section{Analyses}

The COPPAR registry data will be analyzed to answer questions in four priority areas. First, we will examine a longitudinal disease states and progression in patients with $\mathrm{PsO}$ and PsA. This includes stratification by baseline disease phenotype and if available biomarkers and genotypes. Second, care patterns over time will be described, such as health services use, medication use, and use of other medical interventions. This includes comparing individual care patterns regarding their baseline health status and health outcomes after sufficient risk adjustment. Analyses can be stratified by baseline disease phenotype and biomarkers and genotype. Third, we will assess health outcomes of defined disease states and identify how biomarkers predict disease progression. Fourth, the comparative effectiveness of newer immunomodulating medications will be analyzed.

For the preliminary analysis included here, we searched the EMR system of the Brigham and Women's Hospital using the algorithm described above to identify candidate patients for COPPAR. Patients were stratified according to a PsO and PsA diagnosis. For both patient groups we tabulated key demographics, comorbidities, and treatment characteristics as derived from the EMR system. Using the medical record number, we crosschecked whether bio-specimens of these patients were already available in the Dermatology Biobank [42]. For both patient groups we then tabulated the frequency of existing bio-specimens.

\begin{tabular}{|c|c|c|c|c|c|c|c|c|c|}
\hline Visit & B & $\mathbf{i}$ & $F$ & $\mathbf{i}$ & $F$ & $\mathbf{i}$ & $F$ & $\mathbf{i}$ & $\mathbf{F}$ \\
\hline Month & 0 & 3 & 6 & 9 & 12 & 15 & 18 & 21 & 24 \\
\hline Year & 0 & & & & 1 & & & & 2 \\
\hline
\end{tabular}

Fig. 1 Typical follow-up schedule for patients in the COPPAR registry. B: Baseline visit with Informed Consent, Physician Assessment, Patient Questionnaire, labs and sample collection. i: Internet based abbreviated Patient Questionnaires. F: Follow-up visit with Physician Assessment, Patient Questionnaire and labs 
Table 2 Bio-specimens Collection Plan for the COPPAR registry

\begin{tabular}{llll}
\hline Blood Collection Schedule & Baseline & 12 months & 24 months \\
\hline Plasma Blood Monocyte Count & $\checkmark$ & $\checkmark$ & $\checkmark$ \\
Plasma & $\checkmark$ & $\checkmark$ & $\checkmark$ \\
Serum & $\checkmark$ & $\checkmark$ & $\checkmark$ \\
DNA & $\checkmark$ & & \\
RNA & $\checkmark$ & $\checkmark$ & $\checkmark$ \\
Skin Biopsy & $\checkmark$ & $\checkmark$ & $\checkmark$ \\
\hline
\end{tabular}

\section{Data availability}

The registry described in the current study can be inspected jointly with the corresponding author on reasonable request. Upon request, data will be shared with qualified investigators after a material transfer agreement is executed.

\section{Preliminary results}

In our search of the Brigham and Women's Hospital's EMR system, we identified a total of 2484 candidate patients. Of these, 1694 had PsO and 1028 had PsA; 238 patients of the 1694 PsO patients also qualified as having PsA based on their diagnosis coding, indicating a transition from PsO to PsA. The mean age was 55 years, with
$47 \%$ being male. Representative for the Greater Boston area, $85 \%$ of patients were white, $3 \%$ African-American, and $6 \%$ Hispanic. $83 \%$ of the patients had at least one visit for PsO or PsA in 2014. On average they had three clinic visits at the $\mathrm{BWH}$ in a single year. Corresponding to the patients' age distribution, $17 \%$ had diabetes, $38 \%$ hyperlipidemia and $45 \%$ hypertension. The average BMI was 29.6. At the time of identification, $33 \%$ of the patients used prednisone and $10 \%$ used NSAIDs, $86 \%$ used topical psoriasis medications. Among PsA patients the current or prior use of MTX was $60 \%$ and use of TNFi $66 \%$. The use of these medications was lower in PsO patients with MTX by $20 \%$ and TNFi use by $20 \%$ (Table 3 ). Among these patients, we identified a total of 1299 biospecimens already available in the Brigham Biobank. Among PsA patients, $26 \%$ had a DNA sample available, among PsO patients $11 \%$ had DNA available (Table 4).

\section{Discussion}

The aim of the COPPAR registry of patients with PsO and/or PsA was to develop a comprehensive longitudinal data asset that allows researchers to determine predictors of PsA among patients initially presenting with $\mathrm{PsO}$; to identify predictors of treatment response and failure

Table 3 Currently Eligible Patient Population for the COPPAR registry ${ }^{a}$

\begin{tabular}{|c|c|c|c|}
\hline & Psoriasis $(N=1694)^{* *}$ & Psoriatic Arthritis $(N=1028)^{* *}$ & Total Cohort $(N=2484)$ \\
\hline & \multicolumn{3}{|l|}{$N(\%)$ or mean (+/-SD) } \\
\hline \multicolumn{4}{|l|}{ Demographics/Health Services Use } \\
\hline Age, mean, years & $55.5( \pm 16.2)$ & $55.9( \pm 14.2)$ & $55.5( \pm 15.6)$ \\
\hline Male sex & $788(46.5)$ & $494(48.1)$ & $1162(46.8)$ \\
\hline \multicolumn{4}{|l|}{ Race } \\
\hline White & $1382(81.6)$ & $917(89.2)$ & $2099(84.5)$ \\
\hline Black & $61(3.6)$ & $19(1.8)$ & $69(2.8)$ \\
\hline Hispanic & $127(7.5)$ & $23(2.2)$ & $139(5.6)$ \\
\hline Other & $124(7.3)$ & $69(6.7)$ & $177(7.1)$ \\
\hline Patients with visits in 2014 for PsO/PsA & $1404(82.9)$ & $841(81.8)$ & $2068(83.3)$ \\
\hline Visits in 2014 for PsO/PsA, mean & $3( \pm 5)$ & $3( \pm 2)$ & $3( \pm 4)$ \\
\hline \multicolumn{4}{|l|}{ Comorbidities } \\
\hline Diabetes & $318(18.8)$ & $169(16.4)$ & $420(16.9)$ \\
\hline Hyperlipidemia & $740(43.7)$ & $302(29.4)$ & $942(37.9)$ \\
\hline Hypertension & $835(49.3)$ & $422(41.1)$ & $1110(44.7)$ \\
\hline Body mass index, mean & $29.5( \pm 7.7)$ & $29.8( \pm 6.9)$ & $29.6( \pm 7.4)$ \\
\hline \multicolumn{4}{|l|}{ Psoriasis and Psoriatic Arthritis Characteristics } \\
\hline Prednisone use & $470(27.7)$ & $478(46.5)$ & $833(33.5)$ \\
\hline Current NSAID use & $107(6.3)$ & $153(14.9)$ & $248(10.0)$ \\
\hline Current/prior MTX use & $335(19.8)$ & $616(59.9)$ & $801(32.2)$ \\
\hline Current/prior TNFi & $350(20.7)$ & $675(65.7)$ & $858(34.5)$ \\
\hline Current/prior topical PsO & 1625 (95.9) & $728(70.8)$ & $2123(85.5)$ \\
\hline
\end{tabular}

a Based on electronic medical record review

** At least three diagnoses of PsO (ICD-9-CM code 696.1 or ICD-10 code L40.0) or at least three diagnoses of PsA (ICD-9-CM code 696.0 or ICD-10 code L40.5) 
Table 4 Current Bio-specimen repository for the COPPAR registry

\begin{tabular}{llllll}
\hline Blood Collection Schedule & \multicolumn{2}{c}{ Psoriasis } & & \multicolumn{2}{c}{ Psoriatic Arthritis } \\
\cline { 2 - 3 } \cline { 6 - 6 } \cline { 6 - 6 } & $\mathrm{N}$ & $\%$ & & $\mathrm{~N}$ & $\%$ \\
\hline Plasma Blood Monocyte Count & 153 & $9.0 \%$ & & 106 & $6.3 \%$ \\
Plasma & 190 & $11.2 \%$ & & 220 & $13.0 \%$ \\
Serum & 0 & $0.0 \%$ & 33 & $1.9 \%$ \\
DNA & 187 & $11.0 \%$ & 266 & $15.7 \%$ \\
RNA & 91 & $5.4 \%$ & 48 & $2.8 \%$ \\
Skin Biopsy & 2 & $0.1 \%$ & 3 & $0.2 \%$ \\
\hline
\end{tabular}

and characterize treatment transitions; to quantify health services utilization and quality of life of patients with $\mathrm{PsO}$ and PsA; to assess relevant subgroups of patients with specific phenotypes; and to determine the effectiveness and safety of new treatments.

The conception and methodology of COPPAR follows the successful implementation of BRASS (Brigham Rheumatoid Arthritis Studies) that exists since 2003, and is frequently used for research on disease progression and the safety and effectiveness of medical treatment [42-45].

Specific advantages of COPPAR are that it leverages an infrastructure already developed for BRASS, a rheumatoid arthritis registry, that it is a single center cohort with large clinical caseload of interconnected dedicated dermatology and rheumatology practices, that it has access to electronic medical record and an existing bio-repository, that patients receive various pharmacologic strategies allowing for non-randomized comparative effectiveness and biomarker studies, and that patients can be re-contacted by mail or electronically for additional prospective studies (trials, additional biomarkers). The participating physicians and investigators have strong clinical and academic track records in all relevant areas, such as clinical care of $\mathrm{PsO}$ and PsA, biobanking, comparative effectiveness research, pharmacoepidemiology, and patient-reported outcomes measurement. The registry team integrates strong expertise in skin and joint diseases into a single registry.

Recently established, the COPPAR registry is actively recruiting patients with $\mathrm{PsO}$ and $\mathrm{PsA}$ starting May 2017 with the goal to quickly complete a base cohort of $1000 \mathrm{PsO}$ and PsA patients each. These patients will be followed long-term and additional patients will join over time.

\section{Abbreviations}

BASDAl: Bath Ankylosing Spondylitis Disease Activity Index; BSA: Body Surface Area; CASPAR: Classification criteria for psoriatic arthritis; COPPAR: Cohort for Psoriasis and Psoriatic Arthritis Registry; EMR: Electronic medical record; FACIT: Functional Assessment of Chronic Illness Therapy; IPAQ: International Physical Activity Questionnaire; LEl: LEEDS Enthesitis Index; PASI: Psoriasis Areas Severity Index; PBMCs: Peripheral blood mononuclear cells; PGA: Patient Global Assessment, and Physician Global Assessment; PsA: Psoriatic arthritis; PsO: Psoriasis; REDCap: Research Electronic
Data Capture; SARM: Skin and Related Musculoskeletal Diseases; sPGA: Physician Static Global Assessment; TNFi: Tumor necrosis factor alpha inhibitor; VAS: Visual analog scales; WPAl: Work Productivity and Activity Impairment

\section{Acknowledgements}

We like to acknowledge the programming support received for this project by Ye Gou.

\section{Funding}

This study was funded by internal resources of the Division of Rheumatology and the Department of Dermatology, Brigham and Women's Hospital, Boston, MA.

\section{Availability of data and materials}

The datasets used and/or analyzed during the current study can be inspected jointly with the corresponding author on reasonable request. Individual-level data cannot be shared beyond the premises of the Brigham and Women's Hospital.

\section{Authors' contributions}

MS made substantial contributions to conception and design, acquisition of data, analysis and interpretation of data; she has been drafting the manuscript and revising it critically for important intellectual content. JFM made substantial contributions to conception and design, acquisition of data, analysis and interpretation of data; he has been involved in drafting the manuscript or revising it critically for important intellectual content. EWK made substantial contributions to conception and design, acquisition of data, analysis and interpretation of data; he has been involved in drafting the manuscript or revising it critically for important intellectual content. DHS made substantial contributions to conception and design, acquisition of data, analysis and interpretation of data; she has been involved in drafting the manuscript or revising it critically for important intellectual content. All authors read and approved the final manuscript.

\section{Ethics approval and consent to participate}

The COPPAR registry and the resulting research was approved by the institutional review board of the Brigham and Women's Hospital, Boston, MA. The review included the informed consent documents for registry participation and bio-specimens. All study staff are trained in patient privacy and data handling according to HIPAA.

\section{Consent for publication}

Not applicable.

\section{Competing interests}

Maria Schneeweiss has no potential competing interest to declare. Dr. Joseph Merola is a consultant for Biogen IDEC, Eli Lilly, Novartis, and Momenta. He sits on advisory boards for Biogen IDEC, AbbVie, Amgen, Eli Lilly, Novartis, Janssen and Mallinckrodt. He is a speaker for AbbVie and Eli Lilly. He is also an investigator of Biogen IDEC, Amgen, Pfizer, and Boehringer Ingelheim, and has a licensed outcome measure with AbbVie. He has received a grant from Biogen IDEC.

Dr. Daniel Solomon received salary support through research contracts to the Brigham and Women's Hospital from CORRONA, Asta Zeneca, Amgen, Genentech, Pfizer and Lilly. He serves unpaid on the executive Committee for the PRECISION trial, a trial testing the safety of NSAIDs.

Dr. Elizabeth Karlson has no potential competing interest to declare.

\section{Publisher's Note}

Springer Nature remains neutral with regard to jurisdictional claims in published maps and institutional affiliations.

\section{Author details}

${ }^{1}$ Division of Rheumatology, Department of Medicine of the Brigham and Women's Hospital, Harvard Medical School, 75 Francis Street, Boston, MA 02115, USA. Department of Dermatology of the Brigham and Women's Hospital, Harvard Medical School, Boston, USA. ${ }^{3}$ Division of

Pharmacoepidemiology, Department of Medicine of the Brigham and Women's Hospital, Harvard Medical School, 75 Francis Street, Boston, MA 02115, USA. 
Received: 22 January 2017 Accepted: 7 August 2017 Published online: 16 August 2017

\section{References}

1. Gliklich RE, Dreyer N. Registries for evaluating patient outcomes: a User's guide. 2nd ed. Agency for Healthcare Research and Quality: Rockville, MD; 2010.

2. Augustin M, Spehr C, Radtke MA, et al. German psoriasis registry PsoBest: objectives, methodology and baseline data. Journal der Deutschen Dermatologischen Gesellschaft $=$ Journal of the German Society of Dermatology : JDDG. Jan 2014;12(1):48-57.

3. Lofvendahl S, Petersson IF, Theander E, Svensson A, Zhou C, Steen Carlsson K. Incremental Costs for Psoriasis and Psoriatic Arthritis in a Populationbased Cohort in Southern Sweden: Is It All Psoriasis-attributable Morbidity? The Journal of rheumatology. Jan 152016.

4. Khraishi M, MacDonald D, Rampakakis E, Vaillancourt J, Sampalis JS. Prevalence of patient-reported comorbidities in early and established psoriatic arthritis cohorts. Clin Rheumatol. Jul 2011;30(7):877-85.

5. Carneiro JN, Paula AP, Martins GA. Psoriatic arthritis in patients with psoriasis: evaluation of clinical and epidemiological features in 133 patients followed at the University Hospital of Brasilia. An Bras Dermatol. Jul-Aug 2012;87(4):539-44

6. Sarzi-Puttini $P$, Varisco V, Ditto MC, Benucci M, Atzeni F. Psoriatic arthritis registries. The Journal of rheumatology Supplement Nov. 2015;93:30-2.

7. Ogdie A, Weiss P. The epidemiology of psoriatic arthritis. Rheum Dis Clin N Am. Nov 2015;41(4):545-68.

8. Liu JT, Yeh HM, Liu SY, Chen KT. Psoriatic arthritis: Epidemiology, diagnosis, and treatment. World journal of orthopedics. Sep 18 2014;5(4):537-543.

9. Ibrahim G, Waxman R, Helliwell PS. The prevalence of psoriatic arthritis in people with psoriasis. Arthritis and rheumatism. Oct 15 2009;61(10): 1373-1378.

10. Velez NF, Wei-Passanese EX, Husni ME, Mody EA, Qureshi AA. Management of psoriasis and psoriatic arthritis in a combined dermatology and rheumatology clinic. Arch Dermatol Res. Jan 2012;304(1):7-13.

11. Eder L, Gladman DD. Predictors for clinical outcome in psoriatic arthritis what have we learned from cohort studies? Expert Rev Clin Immunol. Jun 2014;10(6):763-70.

12. Azevedo VF, Buiar PG. Risk factors and predictors of psoriatic arthritis in patients with psoriasis. An Bras Dermatol. Mar-Apr 2013;88(2):233-6.

13. Lofvendahl S, Theander E, Svensson A, Carlsson KS, Englund M, Petersson IF. Validity of diagnostic codes and prevalence of physician-diagnosed psoriasis and psoriatic arthritis in southern Sweden-a population-based register study. PLoS One. 2014;9(5):e98024.

14. Asgari MM, Wu JJ, Gelfand JM, et al. Validity of diagnostic codes and prevalence of psoriasis and psoriatic arthritis in a managed care population, 1996-2009. Pharmacoepidemiol Drug Saf. Aug 2013;22(8):842-9.

15. Taylor W, Gladman D, Helliwell P, et al. Classification criteria for psoriatic arthritis: development of new criteria from a large international study. Arthritis Rheum. Aug 2006;54(8):2665-73.

16. Marks R, Barton SP, Shuttleworth D, Finlay AY. Assessment of disease progress in psoriasis. Arch Dermatol. Feb 1989;125(2):235-40.

17. Radloff $L$. The CES-D scale: a self-report de- pression scale for research in the general population. Appl Psychol Meas. 1977;1:385-401.

18. Mease PJ. Measures of psoriatic arthritis: Tender and Swollen Joint Assessment, Psoriasis Area and Severity Index (PASI), Nail Psoriasis Severity Index (NAPSI), Modified Nail Psoriasis Severity Index (mNAPSI), Mander/ Newcastle Enthesitis Index (MEI), Leeds Enthesitis Index (LEI), Spondyloarthritis Research Consortium of Canada (SPARCC), Maastricht Ankylosing Spondylitis Enthesis Score (MASES), Leeds Dactylitis Index (LDI), Patient Global for Psoriatic Arthritis, Dermatology Life Quality Index (DLQI), Psoriatic Arthritis Quality of Life (PSAQOL), Functional Assessment of Chronic IIIness Therapy-Fatigue (FACIT-F), Psoriatic Arthritis Response Criteria (PsARC), Psoriatic Arthritis Joint Activity Index (PsAJAl), Disease Activity in Psoriatic Arthritis (DAPSA), and Composite Psoriatic Disease Activity Index (CPDAI). Arthritis care \& research. Nov 2011:63 Suppl 11:S64-85.

19. Chandran V, Gottlieb A, Cook RJ, et al. International multicenter psoriasis and psoriatic arthritis reliability trial for the assessment of skin, joints, nails, and dactylitis. Arthritis and rheumatism. Sep 15 2009;61(9):1235-1242.

20. Healy PJ, Helliwell PS. Measuring clinical enthesitis in psoriatic arthritis: assessment of existing measures and development of an instrument specific to psoriatic arthritis. Arthritis and rheumatism. May 15 2008;59(5): 686-691.
21. Taylor WJ, Harrison AA. Could the Bath Ankylosing Spondylitis Disease Activity Index (BASDAI) be a valid measure of disease activity in patients with psoriatic arthritis? Arthritis and rheumatism. Jun 15 2004; 51(3):311-315.

22. Langley RG, Ellis CN. Evaluating psoriasis with psoriasis area and severity index, psoriasis global assessment, and lattice system Physician's global assessment. J Am Acad Dermatol. Oct 2004;51(4):563-9.

23. Long CC, Finlay AY, Averill RW. The rule of hand: 4 hand areas $=2 \mathrm{FTU}=1$ g. Arch Dermatol. Aug 1992;128(8):1129-30.

24. Ashcroft DM, Wan Po AL, Williams HC, Griffiths CE. Clinical measures of disease severity and outcome in psoriasis: a critical appraisal of their quality. Br J Dermatol. Aug 1999;141(2):185-91.

25. Feldman SR, Krueger GG. Psoriasis assessment tools in clinical trials. Annals of the rheumatic diseases. Mar 2005;64 Suppl 2:ii65-68; discussion ii69-73.

26. Chow C, Simpson MJ, Luger TA, Chubb H, Ellis CN. Comparison of three methods for measuring psoriasis severity in clinical studies (part 1 of 2): change during therapy in psoriasis area and severity index, static Physician's global assessment and lattice system Physician's global assessment. Journal of the European Academy of Dermatology and Venereology : JEADV. Jul 2015;29(7):1406-14.

27. Husted J, Gladman DD, Farewell VT, Long JA. Validation of the revised and expanded version of the arthritis impact measurement scales for patients with psoriatic arthritis. J Rheumatol. Jun 1996;23(6):1015-9.

28. Yang $Y$, Brazier J, Longworth L. EQ-5D in skin conditions: an assessment of validity and responsiveness. The European journal of health economics: HEPAC : health economics in prevention and care. Dec 2015;16(9):927-39.

29. Finlay AY, Khan GK. Dermatology life quality index (DLQI)-a simple practical measure for routine clinical use. Clin Exp Dermatol. May 1994;19(3):210-6.

30. Nichol MB, Margolies JE, Lippa E, Rowe M, Quell J. The application of multiple quality-of-life instruments in individuals with mild-to-moderate psoriasis. PharmacoEconomics. Dec 1996;10(6):644-53.

31. Healy PJ, Helliwell PS. Psoriatic arthritis quality of life instrument: an assessment of sensitivity and response to change. J Rheumatol. Jul 2008; 35(7):1359-61.

32. Patel M, Liu SW, Qureshi A, Merola JF. The Brigham scalp nail inverse palmoplantar psoriasis composite index (B-SNIPI): a novel index to measure all non-plaque psoriasis subsets. J Rheumatol. Jun 2014;41(6): 1230-2.

33. Cauli A, Gladman DD, Mathieu A, et al. Patient global assessment in psoriatic arthritis: a multicenter GRAPPA and OMERACT study. J Rheumatol. May 2011;38(5):898-903.

34. Zhang W, Bansback N, Boonen A, Young A, Singh A, Anis AH. Validity of the work productivity and activity impairment questionnaire-general health version in patients with rheumatoid arthritis. Arthritis research \& therapy. 2010;12(5):R177.

35. Tillett W. De-Vries C, McHugh NJ. Work disability in psoriatic arthritis: a systematic review. Rheumatology. Feb 2012;51(2):275-83.

36. Chandran V, Bhella S, Schentag C, Gladman DD. Functional assessment of chronic illness therapy-fatigue scale is valid in patients with psoriatic arthritis. Ann Rheum Dis. Jul 2007;66(7):936-9.

37. Hagstromer M, Oja P, Sjostrom M. The international physical activity questionnaire (IPAQ): a study of concurrent and construct validity. Public Health Nutr. Sep 2006:9(6):755-62.

38. Torres T, Alexandre JM, Mendonca D, Vasconcelos C, Silva BM, Selores M. Levels of physical activity in patients with severe psoriasis: a cross-sectional questionnaire study. Am J Clin Dermatol. Apr 2014;15(2):129-35.

39. lannaccone CK, Lee YC, Cui J, et al. Using genetic and clinical data to understand response to disease-modifying anti-rheumatic drug therapy: data from the Brigham and Women's Hospital rheumatoid arthritis sequential study. Rheumatology. Jan 2011;50(1):40-6.

40. Bykerk VP, Shadick N, Frits M, et al. Flares in rheumatoid arthritis: frequency and management. A report from the BRASS registry. J Rheumatol. Feb 2014; 41(2):227-34.

41. Pittman CA, Miranpuri AS. Neurosurgery clinical registry data collection utilizing informatics for integrating biology and the bedside and electronic health records at the University of Rochester. Neurosurg Focus. Dec 2015;39(6):E16.

42. Centola M, Cavet G, Shen Y, et al. Development of a multi-biomarker disease activity test for rheumatoid arthritis. PLoS One. 2013;8(4):e60635. 
43. Lee YC, Frits ML, lannaccone CK, et al. Subgrouping of patients with rheumatoid arthritis based on pain, fatigue, inflammation, and psychosocial factors. Arthritis \& rheumatology Aug 2014;66(8):2006-2014.

44. Cui J, Taylor KE, Lee YC, et al. The influence of polygenic risk scores on heritability of anti-CCP level in RA. Genes Immun. Mar 2014;15(2):107-14.

45. Lillegraven $\mathrm{S}$, Paynter $\mathrm{N}$, Prince $\mathrm{FH}$, et al. Performance of matrix-based risk models for rapid radiographic progression in a cohort of patients with established rheumatoid arthritis. Arthritis care \& research. Apr 2013;65(4):526-33.

Submit your next manuscript to BioMed Central and we will help you at every step:

- We accept pre-submission inquiries

- Our selector tool helps you to find the most relevant journal

- We provide round the clock customer support

- Convenient online submission

- Thorough peer review

- Inclusion in PubMed and all major indexing services

- Maximum visibility for your research

Submit your manuscript at www.biomedcentral.com/submit 